\title{
RSA - QoS: A Resource Loss Aware Scheduling Algorithm for Enhancing the Quality of Service in Mobile Networks
}

\author{
*K. Ramkumar ${ }^{1}$ and P. Calduwel Newton ${ }^{2}$ \\ ${ }^{1}$ Research Scholar, Department of Computer Science, Bishop Heber College (Autonomous) \\ Tiruchirappalli - 620 017, Tamil Nadu, India. \\ [e-mail:kramdharma@gmail.com] \\ ${ }^{2}$ Assistant Professor, Départment of Computer Science, Government Arts College \\ Thiruverumbur, Tiruchirappalli - 620 022, Tamil Nadu, India. \\ [e-mail:calduwel@yahoo.com] \\ *Corresponding author: K. Ramkumar
}

Received February 26, 2018; revised July 7, 2018; accepted July 18, 2018;

published December 31, 2018

\begin{abstract}
Adaptive Multi-Rate Codec is one of the codecs which is used for making voice calls. It helps to connect people who are scattered in various geographical areas. It adjusts its bit-rate according to the user's channel conditions. It plays a vital role in providing an improved speech quality of voice connection in Long Term Evolution (LTE). There are some constraints which need to be addressed in providing this service profitably. Quality of Service (QoS) is the dominant mechanism which determines the quality of the speech in communication. On several occasions, number of users are trying to access the same channel simultaneously by standing in a particular region for a longer period of time. It refers to Multi-user channel sharing problem which leads to resource loss very often. The main aim of this paper is to develop a novel RSA - QoS scheduling algorithm for reducing the Resource Loss Ratio. Eventually, it increases the throughput.The simulation result shows that the RSA - QoS increases the number of users for accessing the resources better than the existing algorithms in terms of resource loss and throughput. Ultimately, it enhances the QoS in Mobile Networks.
\end{abstract}

Keywords: Adaptive Multi-Rate, Voice over Internet Protocol, Resource Block, Throughput, Resource Loss Ratio and Long Term Evolution.

This research was supported by a research grant from the University Grants Commission, National Fellowship for Students of Other Backward Classes (OBC), New Delhi, India. 


\section{Introduction}

LTE is a technology of Fourth Generation [1] network which is an enhanced version of the third generation network. It was launched in the year of 2004 by $3^{\text {rd }}$ Generation Partnership Project (3GPP) [2]. In High Mobility, it provides 100 Mbps and 50 Mbps for downloading and uploading respectively. In Low Mobility, it offers 1 Gbps data transfer speed along with quicker smooth handoff. LTE has $20 \mathrm{MHz}$ wider in channel bandwidth for accessing purpose including control signals. It supports a vast number of multimedia applications [3] like Skype, GTalk, IMO, Online Gaming, etc. The quality of voice has been expanded and enriched in this Fourth Generation network. Circuit Switching has been replaced by Packet switching in order to reduce the connection establishment delay of 4G network. 3GPP has introduced the concept of Voice over Long Term Evolution (VoLTE) [4] to facilitate voice service in LTE. VoIP has become more prominent since it provides voice call in a cheaper cost than VoLTE. It is very sensitive to QoS by having the Quantitative parameters like Delay, Jitter, Packet Loss, Packet Delivery Ratio, Throughput, etc., eventhough, it provides lower cost. eNodeB acts as a scheduler for resource allocation by means of Medium Access Control (MAC) [5]. It plays a reasonable role in assigning resources to the users and terminates the users connection as soon as the service is over. LTE works by invoking the Evolved Universal Terrestrial Radio Access Network (E-UTRAN) architecture for providing the seamless communication. It adapts all types of networks like GSM, CDMA, UMTS, WLAN, etc. Generally, the data transmission is carried out in the form of frames in this network. Fig. 1 shows the structure of the LTE Frame [6] in detail.

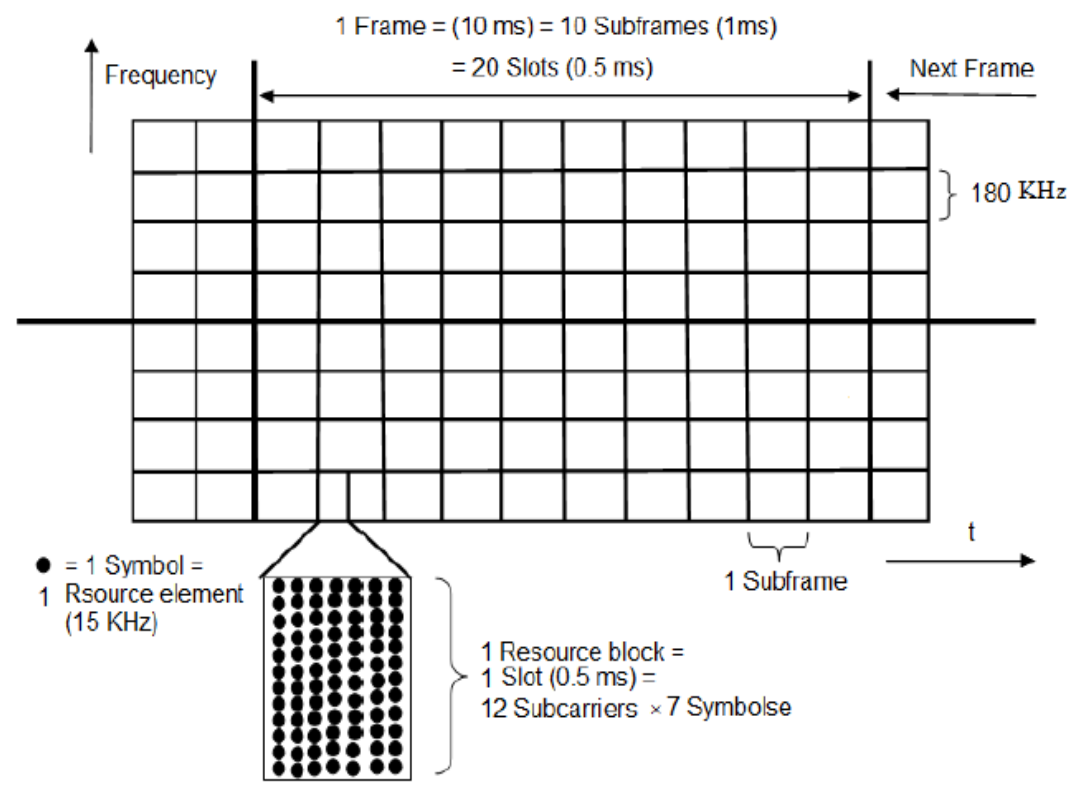

Fig. 1. LTE Frame Structure [6]

One Frame is composed of ten sub frames. Each sub frame lasts for one Transmission Time Interval (TTI). One sub frame is made up of two individual slots where each has $0.5 \mathrm{~ms}$. Based on the channel bandwidth, the number of Resource Blocks differs. One Resource Block is the combination of 12 subcarriers and 7 symbols in normal prefix mode and 6 Symbols in an extended mode. So, totally 1 RB contains 168 (12 X 7 X 2) Resource Elements (RE) or extended contains 144 REs (12 X 6 X 2). Each RE can carry 2, 4 and 6 bits in QPSK, 16 QAM 
and 64 QAM modulations respectively. Mobile users compute and send their CQI values as a feedback to the eNodeB by using the reference symbols. Thereafter, the eNodeB decides the number of resources that, the user can get access for transmission through downlink channel. For downlink scheduling, LTE uses Orthogonal Frequency Division Multiple Access (OFDMA) scheme and Adaptive Modulation and Coding (AMC) adjusts the coding rate for determining the modulation. Fig. 2 explains the flow of LTE downlink scheduling model.

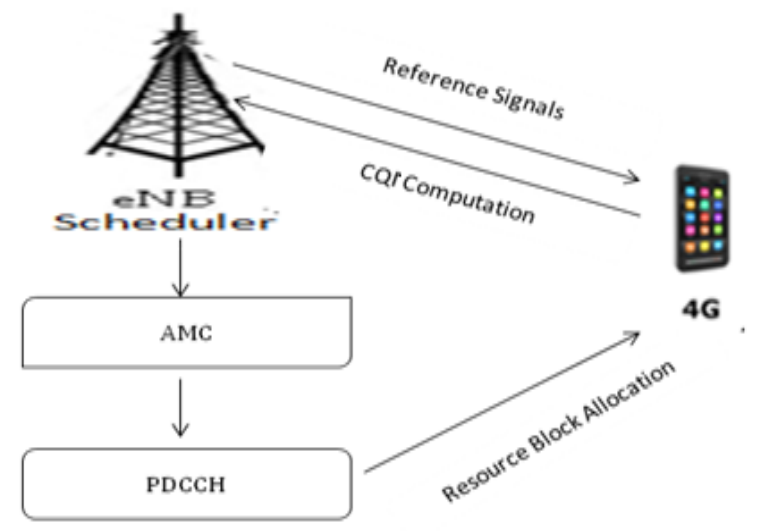

Fig. 2. Downlink Scheduling Model

This paper is structured as follows. Section 2 explains the Review of literature to prepare this article and Section 3 elucidates the proposed RSA - QoS scheduling algorithm to reduce the resource loss in detail. Section 4 discusses the Scenarios and Discussions of the proposed algorithm, Section 5 provides the Mathematical Model and Notations, and Section 6 shows the Performance Evaluation. Section 7 deals with the Conclusion and Finally, References is placed in Section 8.

\section{Related Work}

Initial research on scheduling, the channel bandwidth (Time/Frequency based resources) was not used in effective manner. This section shows the inconsistency of the scheduler pertaining to reduction of the resource loss ratio in increasing the system throughput. Capozzi et al. [7] investigated Heterogeneous network, the preliminary steps to develop a scheduling algorithm, its service architecture and the constraints needed to build a scheduling model. It enlists some research problems in scheduling with possible solutions. Round Robin (RR) [8] algorithm works without considering the channel conditions and assigns the resources to the users by First Come First Served basis. It lacks in two things. First, the QoS requirements of an application and second, their demand in resource allocation. It gives fine results in fairness because all the users get an equal opportunity in accessing the resources.

Best Channel Quality Indicator (BCQI) [9] algorithm keeps on assigning the resources to the users only by looking at the channel conditions of highest quality users. It fails to give the resources to the cell edge users. Therefore, fair allocation in BCQI is very poor when comparing with other scheduling algorithms. The resource allocation decision metric of ' $i$ th, user value is calculated by using the Equation (1).

$$
\mathrm{M}_{\mathrm{i}, \mathrm{k}} \mathrm{BCQI}=\mathrm{r}^{\mathrm{i}} \mathrm{k}(\mathrm{t})
$$


Proportional Fair (PF) [10] scheduler is implemented for maintaining the fairness and using the spectrum efficiently. It considers both the channel quality and users past average throughput for scheduling the user and offers sufficient amount of resources to more number of cell edge users. Equation (2) shows the resource allocation decision metric of ' $i$ 'th' user value by PF algorithm.

$$
\mathrm{M}_{\mathrm{i}, \mathrm{k}} \mathrm{PF}=\mathrm{r}^{\mathrm{i}} \mathrm{k}(\mathrm{t}) / \mathrm{R}^{\mathrm{i}}(\mathrm{t})
$$

In Equation (1), $\mathrm{R}_{\mathrm{i}}(\mathrm{t})$ defines the past average throughput of $\mathrm{i}^{\text {th }}$ user at $\mathrm{t}^{\text {th }}$ interval and $\mathrm{r}_{\mathrm{i}}(\mathrm{t})$ defines the achieved throughput of the $i^{\text {th }}$ user at $n-1^{\text {th }}$ Transmit Time Interval (TTI). RR, BCQI and PF are conventional schedulers in LTE. Duy Huy Nguyen, et al. [11] discussed the MLDWF scheduler by using virtual token and introduced the new factor called Maximum Queue Size (MQS) in order to reduce the packet loss rate. Maximum queue fails to identify the user demand and cannot predict changing the user's data rate. Aswin et al. [12] proposed a new downlink scheduler by introducing two phases which helps to increase the throughput and balance the fairness moderately.

Chiapin et al. [13] developed a delay reduced scheduler that assigns the resources to the real-time users followed by non real-time users. It enhances the QoS of real-time application which is designed to increase the spectral efficiency and also fairness of non real-time applications. In [14] authors introduced Buffer Aware Scheduling algorithm which maximizes the throughput of video applications by fixing the weight based priority. In order to achieve it, buffer size and channel conditions are considered as main parameters. It suffers when greater number of users requesting to get the service in the network. The highest priority is assigned to users who has higher channel condition. It does not check the users demand during the resource assignment.

Nandini et al. [15] compared several scheduling algorithms in WiMAX technology and proposed one cross layer algorithm to increase the throughput of real-time users by assigning the highest priority in which NS-2 has been used in analyzing the results. Mohammad et al. [16] done a comparative study of several downlink scheduling algorithms in finding the strength and weakness of LTE by using the parameters such as mobility speed, frame sizes, number of intervals, etc., to the users. Mohamad et al. [17] proposed an algorithm to increase the average cell throughput with degradation of system fairness. It is an extension of proportional fair algorithm used log based metric for balancing the throughput and fairness. It did not consider demands of the users. Nilam et al. [18] explained proportional fair algorithm and its weakness in offering services to the real-time applications. QoS metric with a proportional fair algorithm classifies the high priority and low priority users for achieving the higher throughput of real-time users.

Dejan et al. [19] developed a novel scheduling algorithm to admit each user at most once in the corresponding locations for enhancing the cell capacity and fairness. Proposed algorithm is compared with MAX C/I algorithm and the results were also discussed. Some of the existing downlink scheduling algorithms are focusing on channel quality alone. It never cares about the user demands which leads to the users with Minimum data rate (real-time) and Maximum bandwidth. The users who are very close to the Base Station access more resources than the users who are at longer distances. It leads to starvation of cell edge users. 
Calduwel et al. [20] developed an algorithm to differentiate each codec user from one another by preparing a ranking order. This ranking increases the throughput and maintains the bandwidth of ongoing calls without any wastage. So, bandwidth efficiency is improved using Analytic Hierarchy Process based Call Admission Control algorithm. Calduwel et al. [21] proposed a priority based scheduling algorithm to reduce the average waiting time of the real-time users in the cellular coverage area. Rebekka et al. [22] developed a GBR algorithm to convince more number of real-time users by setting the bandwidth threshold values. It limits the user satisfaction when more users are crossing the threshold values. It is also noted that, the $\mathrm{PF}$ and BCQI do not consider the user requirements.

Chao He et al. [23] enhanced the QoS of real-time users by giving awareness to each user's QoS requirements independently. It sets a regulating factor for balancing the packet loss ratio with the help of MOS value which is being noted in every iteration of user changes. It is quite difficult to fix the exact threshold value in high mobility scenarios which results to unprediction of application requirements. By setting the few thresholds, this proposed work fails to estimate the user demand which increases the loss ratio and also reduces the throughput. Kanmani et al. [24] introduced blind channel estimation algorithm for reducing the cost value and Bit Error Rate in OFDMA. Generally, several channel estimation algorithms function without having the channel knowledge and its information which lead to resource damage. In [25] authors developed channel estimation algorithm for analysing in mountain environment using the pilot symbols based insertion. It is simulated for COST 207 channel model with 1024 subcarrier and reduces the noise rate. It poorly performs in increasing the throughput. Complexity reduced Least Square channel estimation algorithm is proposed by [26]. It reduces the BER by prefixing the channel conditions along with pilot symbol arrangement.

\section{Reasons for Developing the Proposed Algorithm}

Literatures reveal that, many schedulers are focusing only on the channel conditions, not considering into the user's speed along with their varying data rate. Most of the previous works consider the urgency packets with HoL delay, buffer size and etc., it does not occur at all the times. Whenever the number of users gets increased with same data rate which might result in varying users demand and mobility. It is difficult to predict their need since there is a possibility that multiple channels may have the same channel gain condition with different data rate. Some of the existing algorithms consider only the buffer size and application type.

When it fails to estimate an accurate value of channel condition, the scheduler assigns the resources wrongly, it turns into improper link adaption. Finally, scheduler is unable to find the user demand correctly. In order to offer MIMO based service, base station should receive the correct channel conditions from the user's report. Inaccurate CQI values affect the communication between the Base Station and the User.

Channel condition should be in proper method in order to provide more reliable service. Some of the blind channel estimation algorithms fail to do this. So, there is a need to create scheduler which identifies the user requirement along with mobility prediction, henceforth the proposed RSA - QoS is introduced. The proposed work correctly identifies the variable bit-rate from the user device itself. Therefore, an innovative scheduler is required to reduce the loss ratio as well as increase the number of user's access. In general, every scheduling algorithm should be capable of identifying the user's expectations and their channel quality as well. To address all these issues, the following algorithm is proposed to enhance the QoS by 
reducing the resource loss during resource allocation.

\section{RSA - QOS: A PROPOSED ALGORITHM}

RSA - QoS is developed for assigning the required amount of resources to VoIP users. So that, the users can be increased in number. In order to achieve this, the dynamic prediction is applied in each interval for finding the suitable share of every user by fixing certain limitations. It assigns resources to the users based on the demand by using CQI values to improve system capacity. The users of the network who are using the required resources inside the coverage area through which the total cell capacity can be determined. There are some existing algorithms which assign the resources to the users without considering their application types. Sometimes, Real-time applications may consume a constant amount of bandwidth unlike Non Real-time application takes variable amount of bandwidth frequently.

Input: Number of Users, Number of RBs, Number of Intervals and Types of AMR bit rates Initialization:

$\mathrm{N}_{\mathrm{RB}}=\Phi ; \mathrm{N}_{\mathrm{UE}}=\Phi ;$ Tot_RB $=\Phi ; \mathrm{D}_{\mathrm{UE}}=\Phi ; \mathrm{PD}=20 \mathrm{~ms} ; \mathrm{t}=\Phi ; \mathrm{t}-1=\Phi ;$ bit_rate $=\Phi ;$

In RSA - QoS, the scheduler collects information $N_{U E}$ and $D_{U E}$ to assign the resources to the users. These metrics are used to develop (support) the scheduler which enhances the QoS in Mobile Networks.

Proc RSA_QoS (bit_rate, $D_{U E}$ )

Begin

$$
\begin{aligned}
& T o t \_R B=N_{R B} X P D \\
& \text { for }\left(i \leftarrow 0 ; i<N_{U E} ; i++\right) \\
& \text { for }\left(j \leftarrow 0 ; j<N_{R B} ; j++\right) \\
& U E_{i}=\left[C Q I_{1,1} \ldots \ldots \ldots, C Q I_{N, P}\right] \\
& U E_{i}\left[D_{U E}\right]=(P D X \text { bit_rate })+U E_{i}[\mathrm{OH}] \\
& \text { If ( bit_rate } \left.\left[U E_{i}\right] \text { in ' } t \text { ' time }==\text { bit_rate }\left[U E_{i}\right] \text { in ' } t-1 \text { ' time }\right) \text { Then }
\end{aligned}
$$$$
\text { Set as Static_UE[ }[\text { bit_rate] }
$$$$
\text { Else If (bit_rate }\left[U E_{i}\right]_{\text {in ' ' } t \text { ' time ! = bit_rate }\left[U E_{i}\right]_{\text {in ' }} t-1 \text { ' time }} \text { ) Then }
$$$$
\text { Set as Dynamic_UE }[\text { [bit_rate] }
$$

End If

End for

CALL RB_Assignment(CQI);

End

Proc RB_Assignment(CQI)

Begin

Divide the shares for both Static and Dynamic users according to the utility values Distribute the resources by using the following

for $\left(i \leftarrow 0 ; i<N_{U E} ; i++\right)$ 


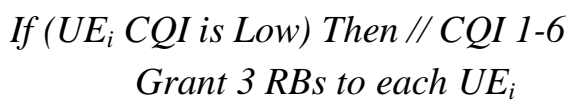

Else If ( $U E_{i} C Q I$ is Med) Then // CQI 7-9

Grant 2 RBs to each $U E_{i}$

Else If (UE $E_{i}$ CQI is High) Then // CQI 10-15

Grant 1 RBs to each $U E_{i}$ End If

End for

End

Output: placing the users one by one according to the channel condition and arrival time. Hence, Resource Loss is reduced by which QoS in Mobile networks can be enhanced. The used mathematical notations and symbols are listed in Table 1.

Table 1. Notations and their Meaning

\begin{tabular}{|c|c|}
\hline Notation & Meaning \\
\hline Bit_rate & AMR codec bit rate \\
\hline $\mathrm{t}, \mathrm{t}-1$ & Current and Previous time of request \\
\hline $\mathrm{N}_{\mathrm{RB}}$ & Number of RBs \\
\hline $\mathrm{N}_{\mathrm{UE}}$ & Number of UEs \\
\hline Tot_RB & Total Number of Available RBs \\
\hline $\mathrm{D}_{\mathrm{UE}}$ & Demand of UE \\
\hline PD & Packetization Delay (20ms) \\
\hline $\mathrm{OH}$ & Overhead in Bytes \\
\hline$C Q I_{N, P}$ & $\begin{array}{c}\text { Channel Quality Indicator in ' } \mathrm{N}^{\text {th, }} \text { Interval } \\
\text { and ' } \mathrm{P}^{\mathrm{th}} \text { ' } \mathrm{RB}\end{array}$ \\
\hline
\end{tabular}

The total number of resources present in the scheduler is depicted in Equation (3). Equation (4) shows that the user CQI calculation for ' $\mathrm{i}^{\text {th' }}$ user which can be repetitively done for all users. Equation (5) explains the ' $\mathrm{i}^{\text {th }}$ ' user's previous $(\mathrm{t}-1)^{\text {th }}$ and current $(\mathrm{t})^{\text {th }}$ bandwidth. Similarly, due to user mobility and/or sudden changes in the network, the user faces congestion from one bandwidth range to another which might either be increased or decreased. In this situation, it is quite difficult to identify the user's demand and scheduler starts assigning the resources poorly. RSA - QoS estimates user needs and offers resources according to their requests. RSA - QoS is developed to allocate the resources efficiently rather than the existing algorithms like ANINTECH, EPF and GBR. RR does not consider user demand which leads to resource saturation. RSA - QoS addresses this issue and assigns the resource blocks in increasing the users in number. It also increases their average throughput and gives more opportunity to the users. It divides the shares equally, even when the user moves from one range into another range while the number of users is less than the other intervals. Generally, the AMR codec produces the speech (audio packets) exactly for every $20 \mathrm{~ms}$ and consider the 
overhead $(\mathrm{OH})$ in bits which is constantly taken by RSA - QoS. It can be applicable to all ranges of AMR codecs in common used for simulation. Most of the codecs would be in $8-15$ CQI range while calculating the total number of bits.

\subsection{User Demand Estimation}

User demand is calculated using the bit-rate and packetization delay. For example, AMR 7.4 bit-rate codec can produce 204 bits (Bandwidth $\mathrm{X} 20 \mathrm{~ms}+\mathrm{OH}=148+56=204$ ) for transmission. If a user is using the AMR codec (4.75 bit-rate) and the value of CQI 10 can be satisfied by giving one RB. According to Table. 2 [27], one RB in QPSK Modulation, the user can transmit 104 bits.

Table 2. Performance of Reference Channel [19]

\begin{tabular}{|c|c|c|c|c|c|c|c|}
\hline Reference Channel & A3-1 & A3-2 & A3-3 & A3-4 & A3-5 & A3-6 & A3-7 \\
\hline Allocated resource blocks & 1 & 6 & 15 & 25 & 50 & 75 & 100 \\
\hline $\begin{array}{c}\text { DFT-OFDM Symbols per } \\
\text { sub frame }\end{array}$ & 12 & 12 & 12 & 12 & 12 & 12 & 12 \\
\hline Modulation & QPSK & QPSK & QPSK & QPSK & QPSK & QPSK & QPSK \\
\hline Code rate & $1 / 3$ & $1 / 3$ & $1 / 3$ & $1 / 3$ & $1 / 3$ & $1 / 3$ & $1 / 3$ \\
\hline Payload size (bits) & 104 & 600 & 1544 & 2216 & 5160 & 6712 & 10296 \\
\hline Transport block CRC (bits) & 24 & 24 & 24 & 24 & 24 & 24 & 24 \\
\hline Code block CRC size (bits) & 0 & 0 & 0 & 0 & 0 & 24 & 24 \\
\hline Number of code blocks -C & 1 & 1 & 1 & 1 & 1 & 2 & 2 \\
\hline $\begin{array}{c}\text { Coded block size including } \\
\text { 12bits trellis termination } \\
\text { (bits) }\end{array}$ & 396 & 1884 & 4716 & 6732 & 15564 & 10188 & 15564 \\
\hline $\begin{array}{c}\text { Total number of bits per } \\
\text { sub-frame }\end{array}$ & 288 & 1724 & 4320 & 7200 & 14400 & 21600 & 28800 \\
\hline $\begin{array}{c}\text { Total symbols per } \\
\text { sub-frame }\end{array}$ & 144 & 864 & 2160 & 3600 & 7200 & 10800 & 14400 \\
\hline
\end{tabular}

Codec based technique [28] adapts this demand for reducing the transmission delay. The RSA-QoS considers some of the AMR-NB Codecs like, 4.75, 6.6, 7.4, 7.95, 8.85 and 10.2 for analysis. A general assumption is made that the users are initially fixed in a position who are moving with little change in speed after a certain time interval. RSA - QoS is applied in all formats of 16 QAM and 64 QAM reference channel parameters to find the number of resource blocks based on the user demand, it assigns the resources to all of them. Other existing algorithms are providing services to less number of users comparing with the RSA - QoS.

CQI value is set to 6 from 1 and fixed a Region of Interest (RoI) value in the simulator for representing all 4.75 Codec users are located in cell edge area. After $20 \mathrm{~ms}$, the users are slowly moving towards base station, some users can access high bandwidth (6.6kbps) and their channel quality could also be high. These changes cause the dynamic mode to be active and user demand keeps fluctuating. It needs to differentiate from one codec type to another which is carried out in the proposed RSA - QoS by changing the mobility model. 


\subsection{RB Assignment}

The RSA - QoS analyses the capacity of various CQIs during transmission of bits and found that, most of the AMR codec can be satisfied by giving one RB in CQI 10 to 15 ranges. So, RSA - QoS assigns one RB to the user who produces CQI value between 10 and 15. Similarly, for other ranges both Low (1-6) and Medium (7-9), RSA - QoS offers 3 RBs, 2RBs respectively. It increases the throughput of cell edge users because of more RBs are assigned to them if they appeared in greater numbers.

\section{Scenarios and Discussions}

Initially, the network scenario is configured by having 10 users, $3 \mathrm{MHz}$ channel bandwidth, 15 RBs, prepared to run for two ms intervals (2TTIs) and shown in Fig. 3. Here, $\mathrm{U}_{1}$ demands 2 RBs in AMR 23.85 codec bit rate, $\mathrm{U}_{2}$ demands 2 RBs in AMR 19.85 codec bit rate, $\mathrm{U}_{3}$ demands $2 \mathrm{RBs}$ in AMR 18.25 codec bit rate, $\mathrm{U}_{4}$ demands $2 \mathrm{RBs}$ in AMR 15.85 codec bit rate, $\mathrm{U}_{5}$ demands $3 \mathrm{RBs}$ in AMR 12.6 codec bit rate, $\mathrm{U}_{6}$ demands $3 \mathrm{RBs}$ in AMR 12.2 codec bit rate, $\mathrm{U}_{7}$ demands $3 \mathrm{RBs}$ in AMR 14.25 codec bit rate, $\mathrm{U}_{8}$ demands $4 \mathrm{RBs}$ in AMR 10.2 codec bit rate, $\mathrm{U}_{9}$ demands 4 RBs in AMR 5.5 codec bit rate and $\mathrm{U}_{10}$ demands 5 RBs in AMR 7.4 codec bit rate in standing at different locations.

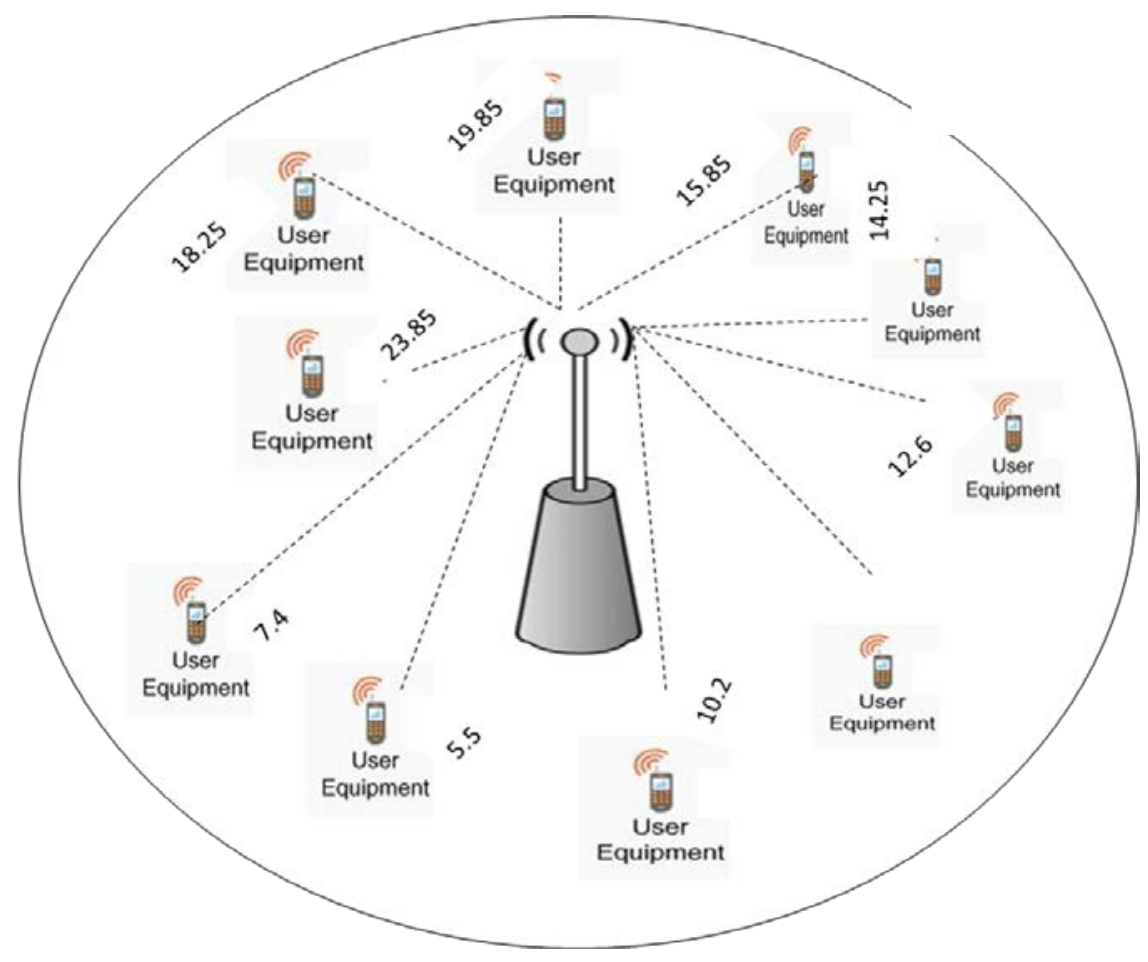

Fig. 3. Example Network Scenario

The total bit transmission (capacity) of each CQI is calculated by using this calculation:1 $\mathrm{RB}=12$ Subcarrier X 14 Symbols $=168$ REs, where PDCCH = 48 REs and Data $=140$ REs. CQI 15 uses 64 QAM, efficiency $=0.948 / 102=0.926,1 \mathrm{RE}=6$ X $0.926=5.5567$, therefore, 120 X $5.5567=666$ bits. It proves that, CQI 15 can carry 666 bits in one RB. An AMR codec 
23.85 bit rate user is having CQI 12 can produce ( $23.85 \mathrm{X} 20 \mathrm{~ms})+56=533$ bits and can be satisfied by having only 2 RBs. The user in CQI 15 can transmit 666 bits and the same user in CQI 12 can transmit 468 bits, therefore for carrying 533 bits 2 RBs are enough. In such a way that, RSA - QoS predicts the demand and grants the resources to the users. Fig. 4 Shows the Resource Loss Ratio Comparison of existing ANINTECH, EPF, GBR and proposed RSA QoS algorithm performance results in detail.

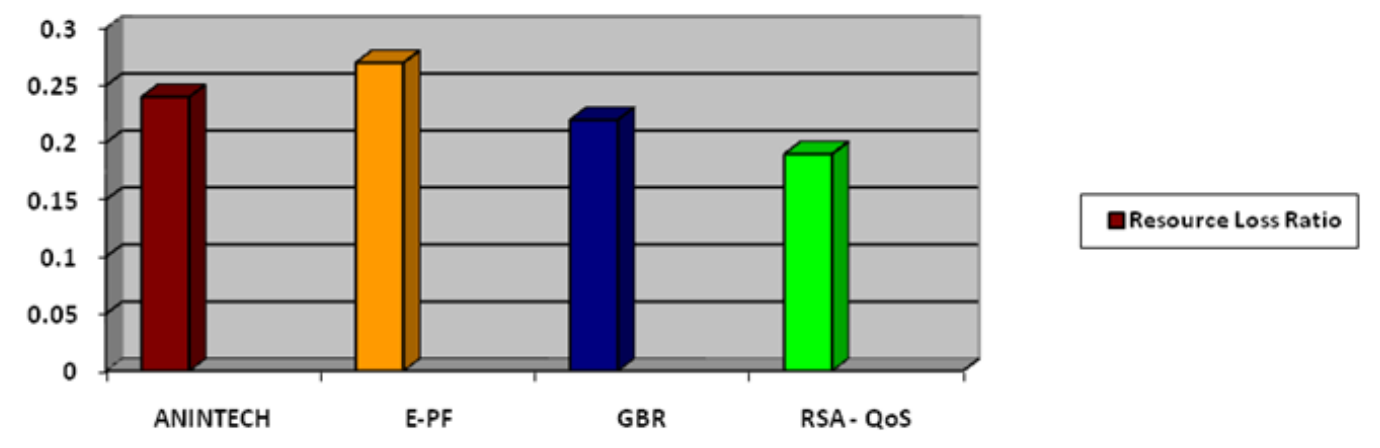

Fig. 4. Resource Loss Ratio Comparisons of Existing and Proposed Algorithms

\section{Mathematical Model and Notations}

The mathematical model of the RSA - QoS algorithm is discussed which adopts set theory. For users, resource allocation and classification of static - dynamic user identification. It concludes the number of resources which can be assigned to each user. The ultimate aim of this model is to bring out the working principle of the proposed algorithm in reducing the resource loss and increasing the throughput.

\subsection{System Model}

The proposed work considers the entire network as Set. It assumes that, set ' $U$ ' $=\left\{u_{1} \ldots u_{n}\right\}$ which contains ' $n$ ' number of elements. Each element of the Set ' $U$ ' signifies a mobile user, where $\mathrm{u}_{1}$ and $\mathrm{u}_{\mathrm{n}}$ denote the first and last elements of the Set respectively. Scheduling is done by collecting the user's feedback in matrix format from each user. Then, the total number of resource blocks is calculated by multiplying the number of available resources per interval with the total number of intervals (i.e. No. of TTI $\times$ No. of Resources per TTI). Equation (6) shows the bandwidth from the first user to the last user and particularly shown for ' $\mathrm{i}$ ' ' user.

$$
\mathrm{u}_{\mathrm{i}}=\{\mathrm{b} \text { for } \mathrm{i}=1 \text { to } \mathrm{n}
$$

where ' $u_{i}$ ' refers to the ' $i$ 'th, user bandwidth

Identify the bandwidth consumption of each user is dynamically changing or not. If all the users use a constant amount of bandwidth which is represented by ' $b$ ' and ' $n$ ' denotes the number of users in the static scenario and defined in Equation (7).

$$
u_{i}=\left\{\begin{array}{l}
b \text { for } i=1 \text { to } r \\
c \text { for } i=r+1 \text { to } n
\end{array}\right.
$$


Equation (7) represents, after the certain interval of time, some of the users may be accessing the variable bandwidth due to its multi-rate feature of codec. Here, few users (1 to r) still are consuming the bandwidth ' $\mathrm{b}$ ' and some other users ( $\mathrm{r}+1$ to $\mathrm{n}$ ) are switching to another bandwidth ' $c$ ' where 'c' refers to the bandwidth of dynamic users and 'b' refers to the bandwidth of static users. Resource balancing decision is done by taking the utility values of both static and dynamic users who are residing in the coverage area. The total resource is equally distributed according to the utility value by applying Equations (8) and (9).

$$
\begin{aligned}
& \text { Static user's share }=\frac{r}{n} \mathrm{mp} \\
& \text { Dynamic user's share }=\frac{\mathrm{n}-\mathrm{r}}{\mathrm{n}} \mathrm{mp}
\end{aligned}
$$

After the share distribution for every user's CQI is taken to grant the number of resource blocks of each user. For example, resource allocation decision is defined in Equation (10).

$$
\text { Resource Block of ' } \mathrm{i}^{\text {th, }} \text { user }=\left\{\begin{array}{l}
1 \mathrm{RB}, \text { if } 10 \leq \mathrm{CQI} \leq 15 ; \\
2 \mathrm{RB} \text {, if } 7 \leq \mathrm{CQI} \leq 9 ; \\
3 \mathrm{RB} \text {, if } 1 \leq \mathrm{CQI} \leq 6 ;
\end{array}\right.
$$

\subsection{Model Comparison}

The corresponding resource block assignment in scheduling is defined by $\mathrm{C}_{\mathrm{ij}}$ variable. It holds the binary relation $\{0$ or 1$\}$ which is shown in Equation (11). The Number of Lost and No Lost users list is shown in Equations (12) and (13).

$$
\begin{gathered}
C_{i j}=\left\{\begin{array}{l}
1 \text { if } i^{\text {th }} \text { user is got assignment to } j^{\text {th }} R B \\
0 \text { if } i^{\text {th }} \text { user is not assigned to } j^{\text {th }} R B
\end{array}\right. \\
\text { Let } R_{L}=\left\{(i, j) / C_{i j}=0\right\} \\
\text { Let } R_{N L}=\left\{(i, j) / C_{i j}=1\right\} \\
\text { Where, } i=1,2, \ldots \text { m and } j=1,2, \ldots n
\end{gathered}
$$

Similarly, the total number of users and their assignment classification can be done by having the Equation (14).

$$
n\left(R_{L}\right)+n\left(R_{N L}\right)=m X n
$$

Where, $n\left(R_{L}\right)$ is the number of users unassigned (i.e. lost) and $n\left(R_{N L}\right)$ is the number of users got assignment. Comparison is shown in Equations (15) and (16) uniquely.

$$
\begin{aligned}
& \mathrm{n}\left(\mathrm{R}_{\mathrm{NL}(\mathrm{P})}\right)>\mathrm{n}\left(\mathrm{R}_{\mathrm{NL}(\mathrm{E})}\right) \\
& \mathrm{n}\left(\mathrm{R}_{\mathrm{L}(\mathrm{P})}\right)<\mathrm{n}\left(\mathrm{R}_{\mathrm{L}(\mathrm{E})}\right)
\end{aligned}
$$

The resource loss ratio comparison of both existing and RSA - QoS is described using the Equation (15) which shows that the RSA-QoS outperforms than the existing algorithm. That is, it is having a greater number of assigned resources to users than unassigned users due to loss ratio. Similarly, RSA - QoS yields less loss ratio than the existing algorithm which is described using the Equation (16). Hence, the RSA - QoS eliminates the users who are wasting the resources frequently and it is proven using the mathematical Equations (15) and (16). 


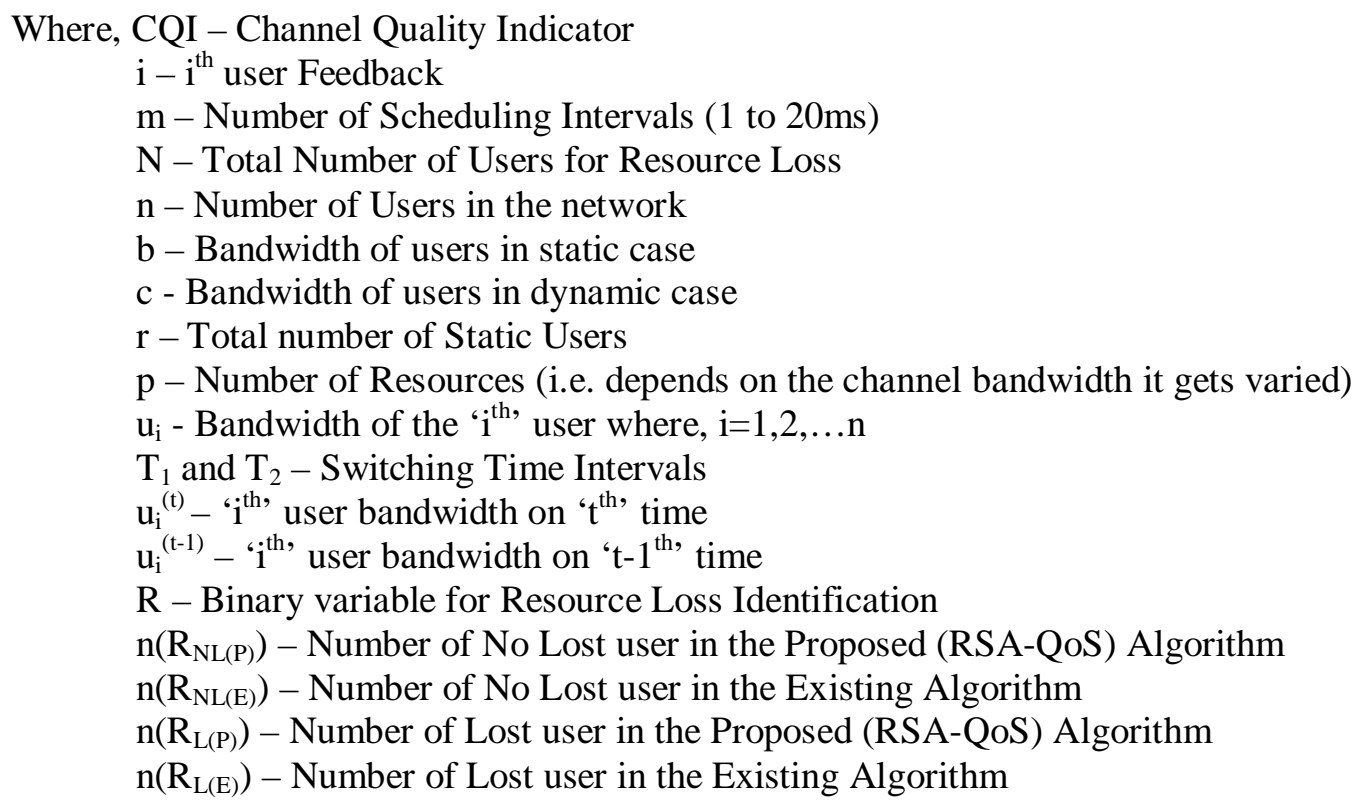

\section{Performance Evaluation}

A Downlink System Level Simulator [29] is used for configuring in both Single-Input Single-Output (SISO) and Multi-Input Multi-Output (MIMO) based network. A Single Base Station with a fixed number of UEs and Mobility management model are taken into consideration for forming the network. Users finite buffer based scenario takes less time whereas, infinite buffer based assumption consumes much time for estimating user's variable demand in short span of time. Initially, the network is configured by taking the parameters such as $1.44 \mathrm{MHz}$ Channel Bandwidth, 20 ms Intervals and 100 Users in Free space path loss model of trial sector tilted environment. At very first time, the UEs are distributed randomly within the cell edge area in simulation. An assumption is made that, every user reports the CQI value between 1 and 6 in each interval. Aftermath, the scheduler assigns the resources by looking at their CQI range and codec bit-rate.

For Case I, each user is assumed to use AMR 4.75 bit-rate codec by having lower CQI values in all the intervals during the resource distribution. Simulation is carried out by choosing the Base Station ' 13 ' and users are placed constantly. In simulation, only the VoIP traffic is enabled by setting ' 1 ' in traffic model method and it is repeatedly done for Five different cases. Existing algorithms are incapable in finding the user demand and misplaces the resources frequently. BCQI offers RBs to the highest channel quality user again and again. It increases the individual user throughput and not the overall throughput. In EPF, an adjustable value is added into the PF and it produces increased average throughput than BCQI. In GBR, the threshold is set by this work then observed the number of users and throughput. ANINTECH offers the resources excessively to only limited number of users and achieves higher throughput in individual users. RSA - QoS shares the resource effectively to 40 users only by assigning $3 \mathrm{RBs}$ and provides better results than the existing algorithms in both rural and urban path loss environments. Table 3 shows the parameters that are used in this simulation of LTE network. 
Table 3. Simulation Parameters and Values

\begin{tabular}{|c|c|}
\hline Parameter & Values \\
\hline Channel Bandwidth & $1.44 \mathrm{MHz}$ \\
\hline Frequency & $2.1 \mathrm{GHz}$ \\
\hline Sector Used & Tri Sector Tilted \\
\hline Base Station & 13 \\
\hline Scheduler & ANINTECH, EPF, GBR and RSA-QoS \\
\hline Simulation TTIs & 20 \\
\hline UEs per eNodeB & 100 \\
\hline Path Loss Models & TS36942, Free Space \\
\hline Path Loss Environment & Urban and Rural \\
\hline Transmit Power & $40 \mathrm{~dB}$ \\
\hline Channel Model & Winner+ \\
\hline UE distribution & Constant UEs per Cell \\
\hline UE speed/Mobility & $15 / 3.6$ (1.38ms) \\
\hline Application Type & VoIP (AMR Codec) \\
\hline
\end{tabular}

In Case II, users in 75\% of QPSK (AMR 4.75 codec) type in the same position and the remaining 25\% of QAM users in 6.6 bit-rate are considered. Also user speed is changed for this mobility. That is, QAM users were moving towards base station that allows for accessing more bandwidth. ANINTECH provides more number of resources to 6.6 codec type user than 4.75 codec users. GBR, EPF gives resources to 4.75 and 6.6 codec users somehow equally. RSA - QoS distributes half amount of resources to both codecs. Case III, users in 50\% of QPSK where using AMR 4.75 codec type, 25\% of the users 16 QAM in AMR 6.6 codec and remaining 25\% of 64 QAM with 6.6 codecs are taken. RSA- QoS gives more resources for 6.6 codec users than others. Case IV, users of $25 \%$ are kept as in AMR 4.75 codec and the rest of the $75 \%$ of the users are in AMR 6.6 codec with 50\% of 64 QAM and 25\% of 16 QAM. More opportunity is given to users who resides near the Base Station in all scheduling algorithms. In this case, RSA - QoS produces more throughput in 6.6 codec rate than other algorithms. Case $\mathrm{V}$ is taken by considering all users in AMR 6.6 codec with 50\% of 16 QAM and 50\% 64 QAM modulations. 95\% of the users got full amount of resources and is given by the RSA - QoS.

ANINTECH changes the priority order and gives more important to VoIP users. For this, it is edited in traffic model and is set to 0.5 , then the rest of the applications are collectively put it as 0.5. It skips more number of users than EPF algorithm because, it assigns all the resources nearby tower users and did not consider both the farthest and medium distance users. Similarly, EPF performs slightly better than ANINTECH when comparing the number of users and an edge user throughput. It produces the higher loss ratio than ANINTECH. Round Robin assigns an equal amount of shares to all of them which performs poorly in throughput for the highest channel quality users. RSA - QoS shows an increased throughput when compared with the other existing algorithms. Table 4 shows the total number of satisfied users in both the existing and RSA - QoS algorithm. 
Table 4. Number of Contented Users in AMR

\begin{tabular}{|c|c|c|c|c|c|c|c|c|c|c|c|c|}
\hline \multirow[b]{2}{*}{$\begin{array}{c}\text { Test } \\
\text { Cases }\end{array}$} & \multicolumn{4}{|c|}{ AMR 4.75 Mode } & \multicolumn{4}{|c|}{ AMR 6.6 Mode } & \multicolumn{4}{|c|}{ Total No. UEs } \\
\hline & $\begin{array}{c}\text { ANIN } \\
\text { TEC } \\
\text { H }\end{array}$ & $\begin{array}{c}\mathbf{E} \\
\mathbf{P F}\end{array}$ & $\begin{array}{c}\text { GB } \\
\mathbf{R}\end{array}$ & $\begin{array}{c}\text { RSA } \\
- \\
\text { QoS } \\
\end{array}$ & $\begin{array}{c}\text { ANIN } \\
\text { TEC } \\
\text { H }\end{array}$ & $\begin{array}{c}\mathbf{E} \\
\mathbf{P F}\end{array}$ & $\begin{array}{c}\text { GB } \\
\mathbf{R}\end{array}$ & $\begin{array}{c}\text { RSA } \\
- \\
\text { QoS } \\
\end{array}$ & $\begin{array}{c}\text { ANIN } \\
\text { TEC } \\
\text { H }\end{array}$ & $\begin{array}{c}\text { E } \\
\text { PF }\end{array}$ & $\begin{array}{l}\mathbf{G} \\
\mathbf{B} \\
\mathbf{R} \\
\end{array}$ & $\begin{array}{c}\text { RSA } \\
- \\
\text { QoS } \\
\end{array}$ \\
\hline $\begin{array}{c}\text { Case I - } \\
\mathrm{T}_{1}(100 \mathrm{UE})\end{array}$ & 23 & 29 & 32 & 40 & - & - & - & - & 23 & 29 & 32 & 40 \\
\hline $\begin{array}{c}\text { Case II - } \mathrm{T}_{1} \\
\& \mathrm{~T}_{2} \\
(75 \mathrm{UE}) \text { and } \\
(25 \mathrm{UE})\end{array}$ & 8 & 14 & 18 & 30 & 17 & 14 & 20 & 15 & 25 & 28 & 38 & 45 \\
\hline $\begin{array}{c}\text { Case III - } \mathrm{T}_{1} \\
\& \mathrm{~T}_{2} \\
\text { (50UE) and } \\
(50 \mathrm{UE})\end{array}$ & 12 & 13 & 22 & 25 & 19 & 25 & 28 & 35 & 31 & 38 & 50 & 60 \\
\hline $\begin{array}{c}\text { Case IV - } \\
\mathrm{T}_{1} \& \mathrm{~T}_{2} \\
(25 \mathrm{UE}) \text { and } \\
(75 \mathrm{UE})\end{array}$ & 9 & 24 & 12 & 10 & 43 & 33 & 50 & 65 & 52 & 57 & 62 & 75 \\
\hline $\begin{array}{c}\text { Case V- } \mathrm{T}_{2} \\
(100 \mathrm{UE})\end{array}$ & - & - & - & - & 71 & 70 & 76 & 95 & 71 & 70 & 76 & 95 \\
\hline
\end{tabular}

\section{Performance Metrics}

The performance metrics which are used to evaluate the proposed RSA - QoS algorithm. RSA-QoS is compared with ANINTECH, EPF and GBR based on the various performance metrics like resource loss ratio and throughput.

\section{Resource Loss Ratio (RLR)}

The Resource Loss Ratio is calculated by taking the ratio of Number of Lost Resources into the Total Number of Received Resources and shown in Equation (17).

$$
\text { RLR }=\frac{\text { No.of Lost Resources }}{\text { Total No.of Received Resources }} \times 100
$$

\subsection{Experimental Results and Analysis}

The RSA - QoS and its comparisons are shown in Fig. 5. and it did not consider the users in High Mobility. Generally, users never wish to make a call while they were in high speed. So that, a little mobility is assumed and applied in the simulator for analyzing the results.

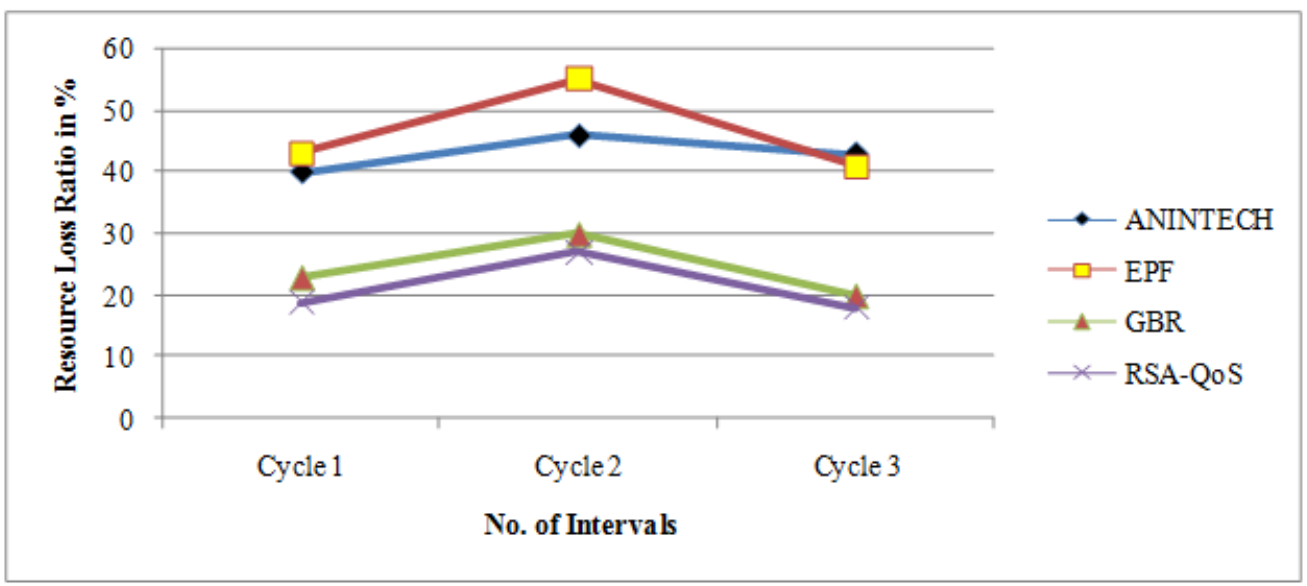

Fig. 5. Resource Loss Ratio Comparison for ANINTECH, EPF, GBR \& RSA - QoS of 100 Users 
RSA-QoS increases the throughput by reducing the resource loss even when channel bandwidth and the number of users get increased. Fig. 6 shows the throughput result of users for 3 cycles. Cycle 1 shows the RB assignment of 40 users, Cycle 2 shows another 40 users and Cycle 3 shows the RB assignment remaining users respectively. In this way, the whole 100 users can get resources appropriately. RSA-QoS is simulated to find the throughput performance of the schedulers in QPSK modulation for 3 cycles.

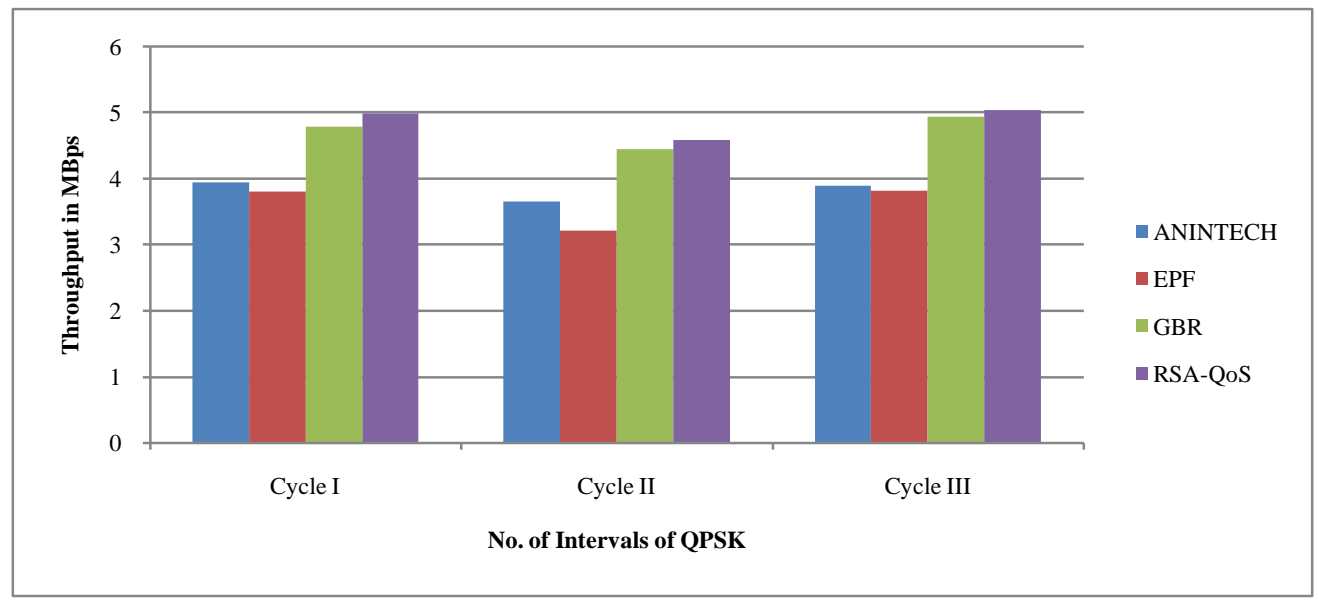

Fig. 6. Throughput Results of ANINTECH, EPF, GBR and RSA-QoS for QPSK

The same setup is used to test in another modulation 16 QAM for different intervals shown in Fig. 7. The throughput is increased gradually because RSA - QoS saves more number of resources.

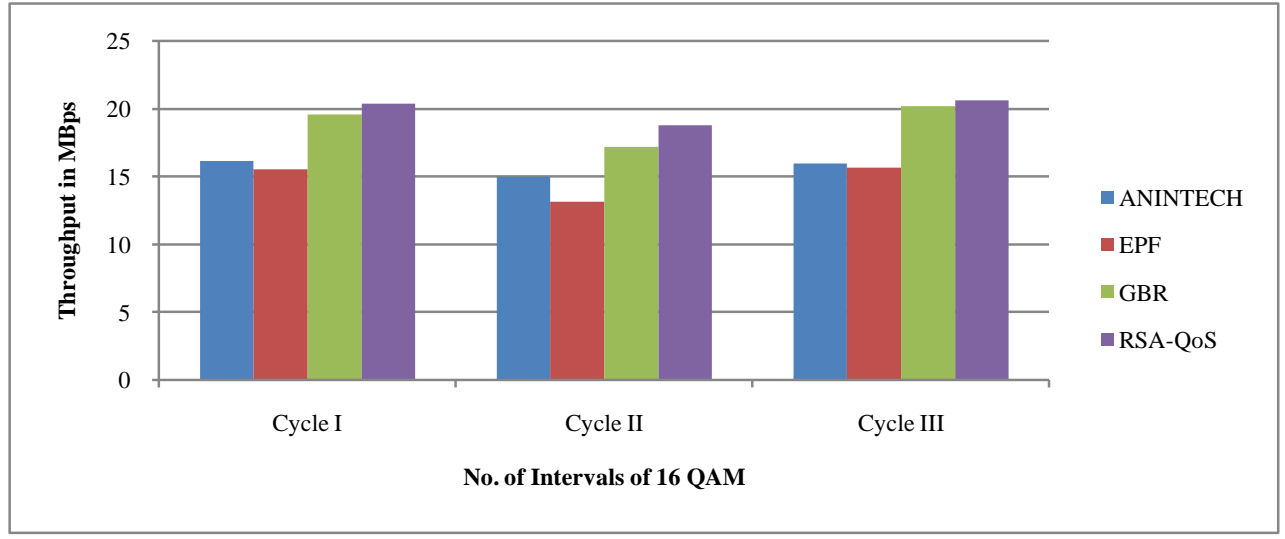

Fig. 7. Throughput Results of ANINTECH, EPF, GBR and RSA-QoS for 16 QAM

In 64 QAM assignment shows more number of user accessing the resource block without losing their intervals with the help of this demand based scheduler. Fig. 8 illustrates the throughput result of same users in 64 QAM. 


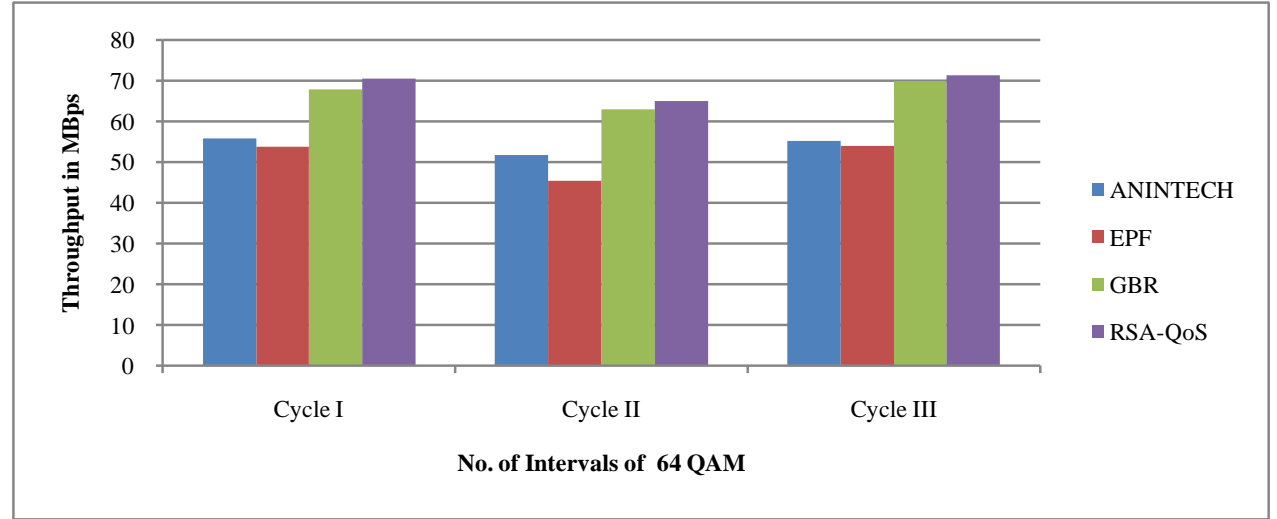

Fig. 8. Throughput Results of ANINTECH, EPF, GBR and RSA-QoS for 64 QAM

The RSA - QoS algorithm keeps on tracking the number of lost resources in every interval for offering to other users in very useful manner. Fig. 9 displays the resource loss ratio performance in variable channel bandwidth, number of RBs in every (5ms) scheduling intervals.

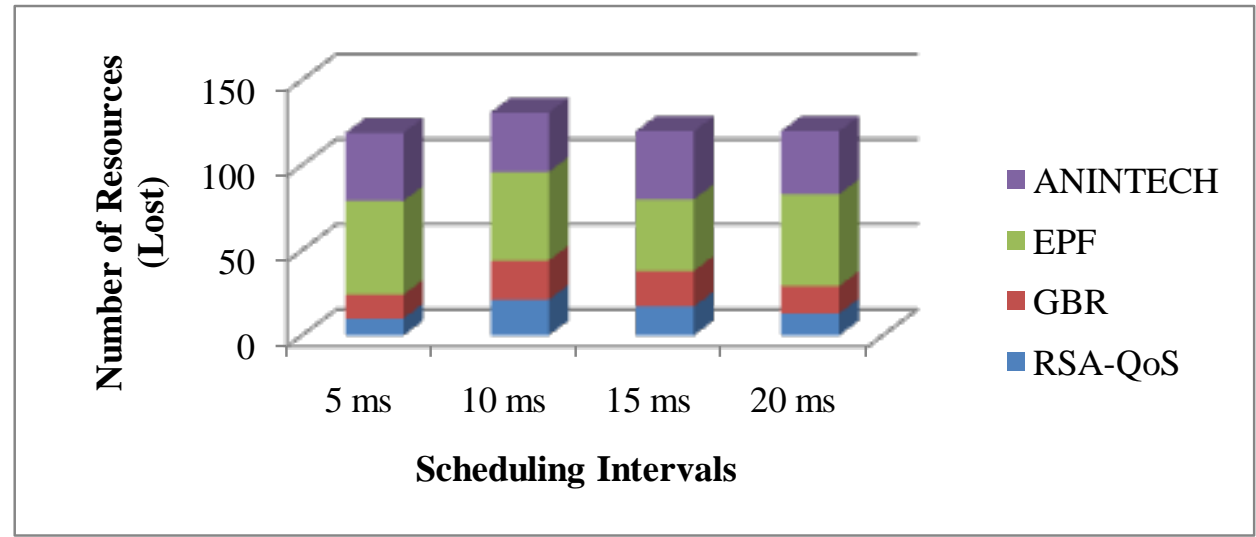

Fig. 9. Resource Loss Ratio for various Intervals

Resource loss ratio is measured by finding the ratio of the total number of missed resource into the total number of received resources of each user. RSA - QoS predicts the user demand and offers the resources according to them.

\section{Conclusion}

In several occasions, the real-time application takes the minimum data rate in transmission and may demand more number of resources with lesser delay residing in a particular sector. It creates a Multi-user channel sharing problem which limits the other user's access, those who are already existed and availed in that service area. If a scheduler assigns the resources to the users without estimating their demand, it would become ineffective. It leads to resource loss during transmission. Resource loss is a traditional issue which drops certain voice messages and finally, receiver can get only half of the message. This problem is addressed by the RSA - QoS algorithm. RSA - QoS allocates the resources to more number of 
users even in various mobility scenarios. In RSA-QoS, High CQI gets only one RB which makes the way to save the resources and assigns them to the low CQI users. User's data rate is predicted at every interval and demand is measured to offer the resources appropriately. It is done by identifying the codec bit rate and its packetization delay of every user. The main aim of this paper is to reduce resource loss ratio and increases the throughput. Ultimately, it enhances the QoS in mobile networks.

\section{References}

[1] E. Dahlman, S. Parkvall and J. Skold, "4G: LTE/LTE-Advanced for Mobile Broadband," 2nd Edition, Elsevier, 2014.

[2] "The Mobile Broadband Standard LTE-Advanced," $3^{\text {rd }}$ Partnership Project 2018, Article (CrossRef Link)

[3] Ying-Hong Wang and Kuo-Feng Huang, "QPPS: QoS Provision Packet Scheduling Algorithm in High Speed Downlink Packet Access," WSEAS Transactions on Communications, Vol. 8, Issue 11, pp. 1160-1169, 2009.

[4] Younes Labyd, Mohammed Moughit, Ab derrahim Marzouk and Ab delkrim Ha qiq, "Performance Evaluation for Voice over LTE by using G.711 as a Codec," International Journal of Engineering Research \& Technology, Vol. 3, Issue 10, pp. 758-763, 2014.

[5] Pallavi Umesh Naik, Shamshad Shirgeri, G.R.Udupi and Plasin Francis Dias, "Design and Development of Medium Access Control Scheduler in LTE eNodeB," International Journal of Computational Engineering Research, Vol. 03, Issue 4, pp. 73-79, 2013.

[6] Mahnaz Sotoudeh Bahreyni and Vahid Sattari Naeini, "Fairness Aware Downlink Scheduling Algorithm for LTE Networks", Journal of Mathematics and Computer Science, Vol. 11, Issue 1, pp. 53-63, 2014. Article (CrossRef Link)

[7] F. Capozzi, G. Piro, L.A. Grieco, G. Boggia and P. Camarda, "Downlink Packet Scheduling in LTE Cellular Networks: Key Design Issues and a Survey,” IEEE Communications Surveys \& Tutorials, Vol. 15, Issue 2, pp. 678-700, 2013. Article (CrossRef Link)

[8] Zuber Patel and Upena Dalal, "Design and Implementation of Low Latency Weighted Round Robin (LLWRR) Scheduling For High Speed Networks," International Journal of Wireless \& Mobile Networks, Vol. 6, No. 4, pp 59-71, 2014. Article (CrossRef Link)

[9] Nsiri Bechira, Mallouki Nasreddinea, Ammar Mahmouda, Hakimi Walida and Mhatli Sofiena, "Novel Scheduling Algorithm for 3GPP Downlink LTE Cellular Network," Procedia Computer Science, Vol. 40, Elsevier, pp. 116-122, 2014. Article (CrossRef Link)

[10] M. H. Habaebi, J. Chebil, A.G. Al-Sakkaf and T. H. Dahawi, "Comparison between Scheduling Techniques In Long Term Evolution,” IIUM Engineering Journal, Vol. 14, Issue 1, pp. 67-76, 2013. Article (CrossRef Link)

[11] Duy Huy Nguyen, Hang Nguyen and Eric Renault, "E-MQS : a new downlink scheduler for real-time flows in LTE network," in Proc. of VTC FALL 2016, IEEE Computer Society, pp.1 - 5, 2016. Article (CrossRef Link)

[12] Aswin Kanagasabai and Amiya Nayak, "Channel Aware Scheduling Algorithm for LTE Uplink and Downlink," Network Protocols and Algorithms, Vol. 7, No. 3, pp. 111-139, 2015. Article (CrossRef Link)

[13] Chiapin Wang and Yuan-Chieh Huang, "Delay-scheduler coupled throughput-fairness resource allocation algorithm in the long-term evolution wireless networks," IET Communications, Vol. 8, Issue 17, pp. 3105-3112, 2014. Article (CrossRef Link)

[14] Meng-Hsien Lin and Yen-Wen Chen, "Performance Analysis of Buffer Aware Scheduling for Video Services in LTE Network," KSII Transactions on Internet and Information Systems, Vol. 9, No. 9, pp. 3594- 3610, 2015. Article (CrossRef Link)

[15] R. Nandini and N. Devarajan, "Comparison For WiMAX Scheduling Algorithms And Proposal Quality Of Service Improvement In WiMAX Networks," American Journal of Applied Sciences, Vol. 11, Issue 1, pp. 8-16, 2014. Article (CrossRef Link) 
[16] Mohammed A. Suliman and Saad Ahmed Ayoob, "A Comparison Study between the Downlink Packet Scheduling Algorithms in LTE Networks," Al Rafidain Engineering, Vol. 23, No. 3, pp 2740, 2015.

[17] M.I. Elhadad, El-Sayed M. El-Rabaie and M. Abd-Elnaby, "Enhanced PF Scheduling Algorithm for LTE Downlink System,” Mobile Computing, Vol. 3, No. 2, pp. 7-12, 2014.

[18] Nilam Dhameliya, Rajni Bhoomarker and Sameena Zafar, "Quality of Service Parameter Centric Resource Allocation for LTE-Advanced,” International Journal of Research in Engineering and Technology, Vol. 03, Issue 02, pp. 330-333, 2014. Article (CrossRef Link)

[19] Dejan Talevski and Liljana Gavrilovska, "Novel Scheduling Algorithms for LTE Downlink Transmission,” Telfor Journal, Vol. 4, Issue 1, pp. 20-25, 2012. Article (CrossRef Link)

[20] P. Calduwel Newton and K.Ramkumar, "TACA- Throughput Aware Call Admission Control Algorithm for VoIP users in Mobile Networks," Advances in Computer and Computational Science, Springer, ISBN: 978-981-10-3770-2, Vol.1, pp. 259-270, 2017. Article (CrossRef Link)

[21] P. Calduwel Newton and K.Ramkumar, "An Innovative Technique to Reduce Delay of VoIP Downlink Packet Scheduling in 4G Technology,” International Journal of Applied Engineering Research, Vol. 10, Issue 72, pp. 363-368, 2015.

[22] B. Rebekka, S. Sudeep and B. Malarkkodi, "An Optimal and Priority Based Rate Guaranteed Radio Resource Allocation Scheme for LTE Downlink," Wireless Personal Communication (Springer), Vol. 83, Issue 3, pp 1643-1661, 2015. Article (CrossRef Link)

[23] Chao He and Richard D. Gitlin, "User-Specific QoS Aware Scheduling and Implementation in Wireless Systems," in Proc. of IEEE 25th International Symposium on Personal, Indoor and Mobile Radio Communications, pp. 1-5, 2014.

[24] M. Kanmani and M. Kannan, "Blind channel estimation for Multiple-Input Multiple-Output system using Constant Modulus Algorithm," Computers and Electrical Engineering, Vol. 44, pp. 42-50, 2015. Article (CrossRef Link)

[25] LinchaoYang, RicaiTian, MinJia and Feng Li, "A Modified LS Channel Estimation Algorithm for OFDM System in Mountain Wireless Environment,” Procedia Engineering, Vol. 29, pp. 2732 2736, 2012. Article (CrossRef Link)

[26] Jianchao Xie, Lihua Yang and Shixiang Shao, " A Low Complexity Channel Estimation Algorithm for Massive MIMO System," in Proc. of 10th International Conference on Communications and Networking in China, pp. 702 - 707, 2015. Article (CrossRef Link)

[27] 3rd Generation Partnership Project (3GPP) Technical Specification, 136141 V8.7.0 (2010-07).

[28] Jeong Wook Seo, Se Jeong Woo and Keun Sung Bae, "A Study on the Application of an AMR Speech Codec to VoIP,” IEEE, pp. 1373 - 1376, 2001. Article (CrossRef Link)

[29] Rupp, Markus, Schwarz, Stefan, Taranetz and Martin, "Vienna LTE-A Simulators", TechnischeUniversitat Wien, Vienna, retrieved from, 2016. Article (CrossRef Link) 


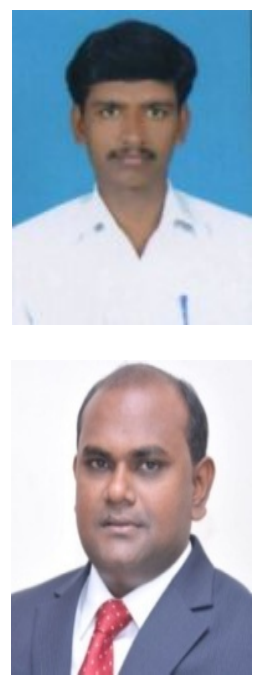

Ramkumar Krishnamoorthy received his Master of Philosophy in Computer Science from Bharathidasan University, Tiruchirappalli, Tamil Nadu, India on 2013. Currently, he is pursuing Doctor of Philosophy in the field of Mobile Networks. He has received financial support from University Grants Commission (UGC) for his research work. He has presented 16 papers including in both National and International Conferences/Journals. His research interests are Quality of Service in Mobile Networks and Network Security.

Calduwel Newton Pitchai is working as an Assistant Professor of Computer Science at Government Arts College, Thiruverumbur, Tiruchirappalli, Tamil Nadu, India. He has published more than 100 papers in the International Journals (Refereed) and Conference Proceedings. He is an active researcher and has presented research papers at Malaysia, Korea, Australia, Sri Lanka and China. He has delivered technical lectures and motivational lectures in various institutions. His research interests are: Wireless Mobile Networks, Quality of Service, Route Optimization and other related topics. 